\author{
Wen-Jei Yang and A. Nouri \\ Department of Mechanical Engineering \\ and Applied Mechanics \\ University of Michigan, Ann Arbor, Michigan 48109
}

(Commmicated by J.P. Hartnett and W.J. Minkowycz)

\begin{abstract}
Surface tension/temperature driven interfacial turbulence in evaporating minute drops was studied photographyicaliy using the laser shadowgraphy. Liguids of relatively low boiling point were employed: ethyl ether, acetone, methanol, carbon tetrachloride, benzene and heptane. A drop surface on a glass plate at room temperature was photographed straight down with motion picture. During drop lifetime, the interface exhibited a dominant pattern of radial stripes over a very brief duration followed by polygonal cells and ended in ripples. These interfacial forms are related to three stages of drop evaporation. The mechanism of interfacial turbulence is proposed.
\end{abstract}

\title{
Introduction
}

The occurence of spontaneous, highly localized, interfacial agitation accompanying mass transfer was first reported by ward and Brooks [1] in 1952. This phenomenon known as interfacial turbulence can cause an order-of-magnitude increase in mass transfer processes such as gas absorption, liquid-liquid extraction and evaporation of mixtures on flat or curved interfaces. Sternling and scriven [2] treated the phenomenon as an evidence of hydrodynamic instability, analyzing a simplified, two-dimensional model (roll cells) by means of the theory of linearlized stability coupled with the hydrodynamic and mass diffusion principles. The 
model assumed that under favorable conditions, small, everpresent fluctuations of concentration or temperature about the interface may be amplified into fully developed flows spontaneously, through the Marangoni effect. Such flow may either be of a highly irregular pattern corresponding to interfacial turbulence or of a cellular pattern called cellular convection. The theory developed the conditions for the initiation of interfacial activity and determined the nature of the dominant disturbance, predicting its wave length, amplification factor, speed of propagation, and temporal period. Using schlieren photography, orell and westwater $[3,4]$ followed the development of polygonal cells which changed to stripes and later evolved into ripples. Polygonal cells and stripes are similar to those observed by Benard for heat transfer. However, the ripples are unexpected from the Sternling-Scriven theory. Later, Ellis and Biddulph [5] observed vigorous rippling on the surface of water during the absorption of acetone or methanol vapor. Using a capacitance probe, they measured wave amplitudes of up to $0.2 \mathrm{~mm}$, proportional to the concentration gradient. Instantaneous surface velocities about $10 \mathrm{~cm} / \mathrm{sec}$ occurred over distances of $1 \mathrm{~cm}$, penetrating $1 \mathrm{~mm}$ into the liquid.

In some chemical absorption processes (gas absorption accompanied by a simultaneous chemical reaction), interfacial turbulence produced by surface tension gradients causes a substantial increase in the rate of gas absorption [6]. Ruckenstein and Berbente [7] determined the conditions under which the Marangoni effect produces interfacial turbulence in the case of mass transfer accompanied by chemical reaction.

In case mass transfer takes place across rounded interfaces, disturbances of the same sort occur. The interface is, in many cases, disturbed by rippling, while the adjoining liquid is turbulently agitated [for example 8,9]. At times, there are localized eruptions at the interface. If it is pendent, the entire drop often pulsates violently and erratically; unattached drops behave in the same way.

In temperature-driven flows, surface-tension effects can cause instability in thin liquid layers, for example [10-13]. 
Reference 13 reveals three basic structural forms of flow in pools of liquids evaporating at room temperature (the fourth pattern, vermiculated rolls, appears only in contaminated surface):cellular network, "ribs" and "streamers" for depths of $2 \mathrm{~mm}$ or less, 3 to $5 \mathrm{~mm}$ and over 7 or $8 \mathrm{~mm}$, respectively.

Kenning [14] pointed out that random surface motion during evaporation of thick liquid layers induced by local variations in temperature would become more pronounded when associated with changes in composition.

The evaporation of liquid drops on a heated surface has important applications to spray cooling, liquid fuel drop combustion, etc. Research efforts have been concentrated on the determination of either drop lifetime [for example 15] or heat transfer rate [for example 16, 17]. Earlier studies indicate moderate to insignificant contribution of the Marangoni effect to heat transfer inside a drop during condensation [18] and in the liquid.surrounding a bubble [19] excluding interfacial instability. Recently, the problem of thermal instability in drops has begun to attract attention [20]. By employing a linear stability theory, reference 20 developed the criterion for the onset of surface-tension induced cellular motion in a hemispherical drop on a solid surface. However, interfacial turbulence of the Marangoni type in drop evaporation has not yet been fully explored. Like in the afore-mentioned mass transfer cases, such localized stirring at the interface would undoubtly lead to high heat transfer coefficients accompanied by high rates of interfacial phase change.

The present study is to explore the nature and pattern of the interfacial activity of minute liquid drops evaporating on a flat glass plate.

\section{Experimental Apparatus and Procedure}

The interfacial activity in the evaporating drops was observed using a conventional shadowgraphic technique, which permits us to see and photograph second derivatives of refractive 
index (and hence second derivatives of temperature) in a transparent medium. The principles of laser illumination and filtering and the method of shadowgraphy have been described in great detail in references 21 and 22-23, respectively.

The shadowgraphic arrangement consisted of an optical system, a test object, and a photographic equipment, as shown in Fig. 1. The test object was a transparent medium, namely a minute drop of evaporating liquid, on an optically flat glass plate, $102 \mathrm{~mm} \times 127 \mathrm{~mm}$ in size and $3 \mathrm{~mm}$ thick. The optical system consisted of five components: light source, achromatic objective, pinhole, a pair of surface mirrors and collimating lens.

A C.W. Radiation Model SP $2,2.5 \mathrm{~mW}$ cylindrical helium-neon laser was used as the light source. The $20 \mathrm{x}$ achromatic microscopic objective was mounted on a frame together with the 10-micron precision pinhole. The collimating lens was placed at its focal length from the pinhole. These four components were aligned on a horizontal bench. Invariably a laser beam contains diffraction noise, flashlamp, or plasma illumination and transverse mode

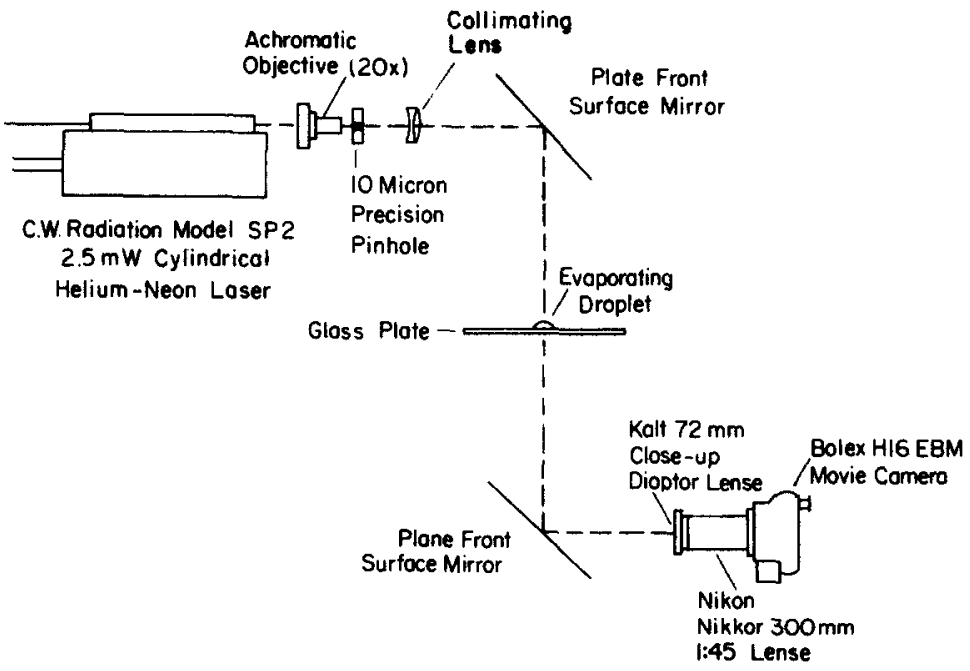

FIG. 1

Achematic of the Shadowgraphic System for Fhotographic Study of Minute Drops Fvaporating on a Solid Surface 
structure. Much of this can be removed by a procedure of spatial filtering-the beam is focused by a high quality lens, the objective, through the tiny pinhole used to control apertures of laser beams and as a spatial filter. The parallel beam of light emerging from the lens was made to pass through the evaporating drop from top to bottom (thus producing a top view of the interfacial activity) by an arrangement of two plate front surface mirrors, $102 \mathrm{~mm} \times 127 \mathrm{~mm}$ and $6 \mathrm{~mm}$ thick, as shown in Fig. 1. The two mirrors are aluminized on surface nearest objects to be viewed so that light may be reflected with no refraction. They were mounted in parallel on a specially-designed frame at 45 degrees. The test plate was inserted into the vertical light beam between the two mirrors. The mirror arrangement was used to obtain a horizontal view of the evaporating drop. The optical path from the lower mirror was horizontal into a 16-mm Bolex H16 EBM movie camera

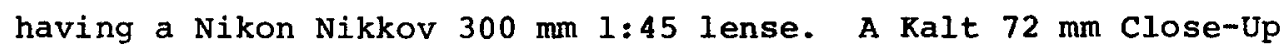
Dioptor lense was mounted on top of the Nikon lense to photograph the air-liquid interface of an evaporating drop. A drop was carefully placed on the test plate by means of a $1-\mu l$ Monoject micro syringe with a bent needle. The needle tip was touching the plate surface before and during the injection to permit the formation of a calm, unsplashing drop.

\section{Photographic Observations}

Various liquids of low boiling point were studied including ethyl ether, acetone, methanol, carbon tetrachloride, benzene, heptane and water. During the course of evaporation, the interface exhibits a variety of geometrical patterns, depending upon mainly the initial size and to a much lesser degree on the physical properties of the liquid. In general, three basic patterns are observed: radial stripes, polygonal cells, and ripples.

Radial stripes - This pattern is first to appear on the interface as soon as the drop is released from a needle tip. All stripes radiate from the surface outward to the drop periphery, as shown in Fig. 2(a). It exists very briefly and the pattern undergoes rapid changes into a cellular structure. The stripes represent "cold lines" where cool fluid would plunge in sheets from the surface into the interior. Morphogically, they are ridges. 


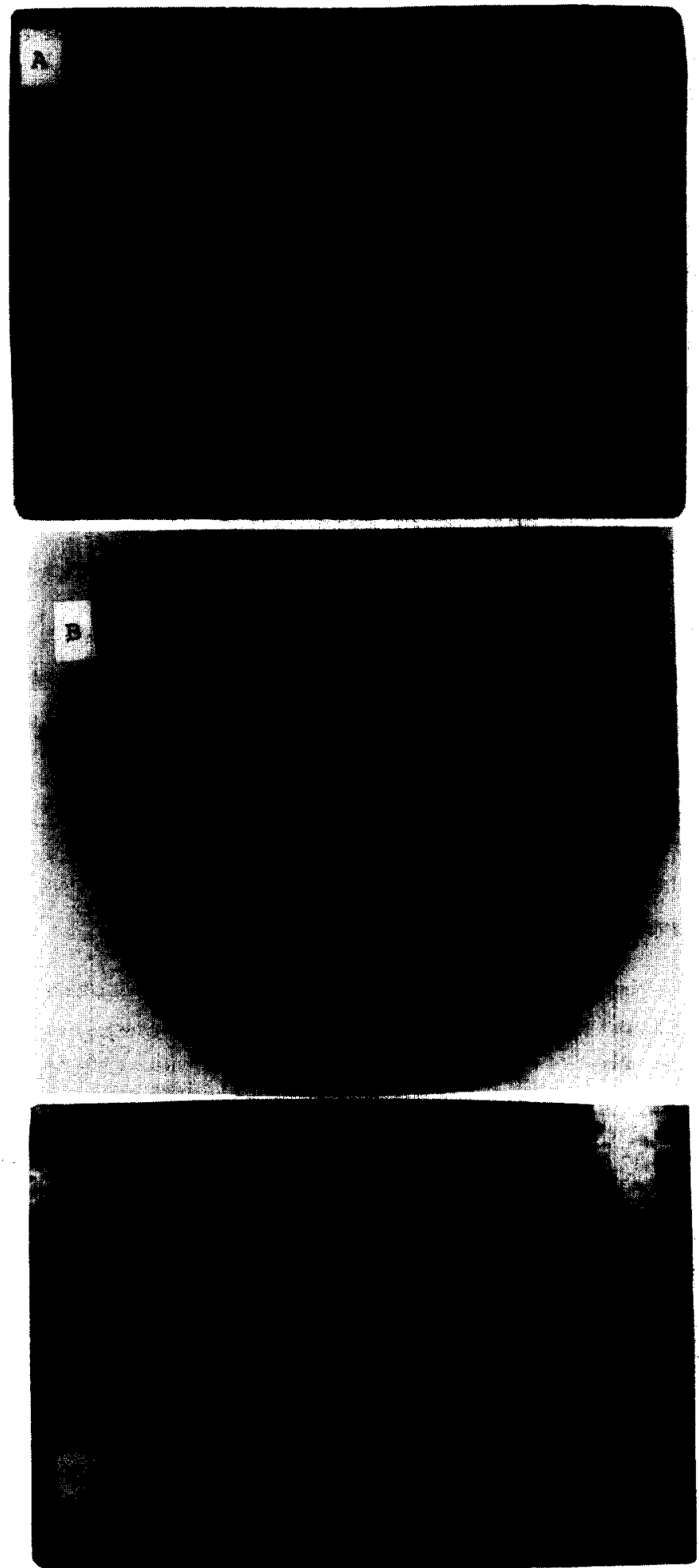

D

$\mathrm{E}$

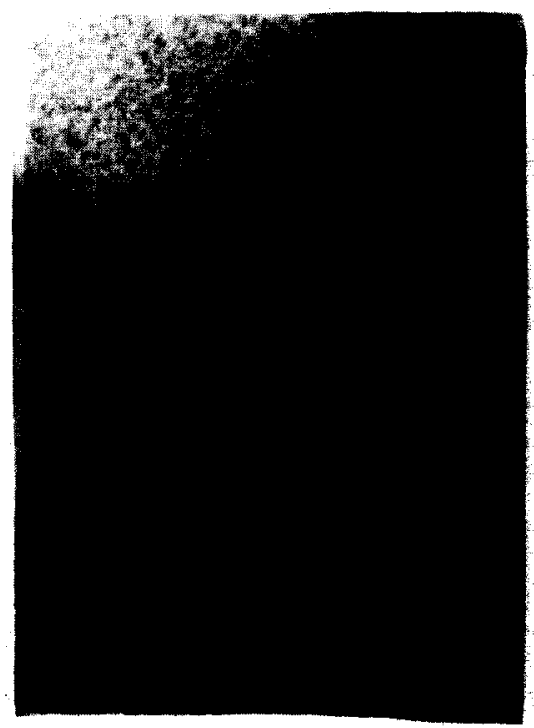

FIG. 2

Typical Interfacial Flow Patterns in Minute Drops 
Polygonal Cells - This pattern usually does not encompass the whole surface, leaving some regions bare and inactive as brigth areas in Fig. 2 (b). The dark oval background represents the laser light. The cells froming the network are mostly irregular polygons with cell partitions well defined. Warm fluid from the interior rises to the surface at each cell center and returns to the interior along the cell partitions as cooled liquid. Each is a Benard cell constituting a small torus independent of the other cells. As time passes, the drop thickness reduces, particularly more rapidly along the drop periphery within which the liquid forms a ring of very thin film. Very closely spaced fine concentric ripples are observed in the very thin liquid film. The width of the ring grows with its innner periphery moving radially inward faster than the retreating of the outer periphery as the drop continues to evaporate. Meanwhile, the thickness of the drop proper within the ring grows thinner and thinner accompanied by a violent variation of the cellular patterns in size and shape.

Ripples - In the final stage of drop evaporation, unconfined ripples grow rapidly inward taking over the area occupied by cellular patterns, like a flood. Often the process proceeds so fast, leaving the traces of large cellular patterns superimposed on the background of unconfined ripples as shown in Fig. 2 (c). Depending on the initial shape of the drop, sometimes, a perfect concentric form of ripples is observed, Fig. 2(d). Its periphery rapidly retreats inward until the completion of liquid evaporation Ridges appear as darkened lines corresponding to "cold lines" at lower temperatures, while troughs emerge as bright lines represent. ing "hot lines" at higher temperature.

The above-mentioned anomalous behavior of water disclosed in the case of pool evaporation [13] was also observed in the present study. As shown in Fig. $2(e)$, the motion picture recorded no trace of interfacial activity in the evaporating drop of various minute size at room temperature. The fluid is characterized by higher thermal conductivity and viscosity which, during the preconvection period are capable of damping and destroying small thermal and mechanical disturbances such that no fluid motion results. From the viewpoint of thermocapillary effect, water has substantially higher surface tension and relatively low surface 
tension-temperature gradient. This combined feature discourages the occurence of interfacial activity. On the other hand, substantially low value of the coefficient of thermal expansion and high kinematic viscosity are not favorable in causing convective flow by the buoyancy driving mechanism in the case of large drops.

\section{Discussion of Results}

Very vigorous and irregular instabilities occur during the evaporation of a drop on a solid surface. The random motion of warm liquid toward the interface to supply the heat for evaporation results in turbulence thus reinforcing the rate of evaporation. Unconfined ripples on the interface are observed in the final stage of drop lifetime. A mechanism is proposed to explain the cause of such an interfacial turbulence.

Since the surface tension is temperature dependent, preferential phase change will give rise to surface tension difference between adjacent slots (lines) or spots (points) in the liquid interface. Slots of preferential evaporation will have a lower surface tension than the neighboring interface liquid and will tend to spread. Sternling and Scriven [2] described these slots or spots as "small, ever-present disturbance" of concentration or temperature about the interface in their hydrodynamic instability theory of interfacial activity. The driving force is the temperature difference between the plate surface and the ambient. Low driving forces produce small surface tension differences. Consequently interface slots will spread slowly causing slight rippling which decays eventually. As the driving force and the rate of preferential evaporation increase, the surface tension gradient increases resulting in greater rippling. At some critical driving force, the rate of evaporation is strong enough to instantaneously produce slots of very steep surface tension gradient. The resulting spreading is so rapid that the momentum of the spreading liquid is sufficient to break the low surface tension slot, thus exposing subjacent liquid drawn from below the interface. A pair of ridges (or an annulus in the case of spot) of low surface tension are then formed and expand outward, leaving behind a region of high surface tension which tends to reverse the spreading motion. This causes liquid to rush from the bulk 
and from the spreading film toward the high surface tension region, resulting in a build up of liquid into another ridge (or a large ripple in the case of spot). Finally, both "hot lines" (warm troughs) and "cold lines" (cold ridges) both of which are called "streamers" are formed in the interface. The sequence of events is illustrated in Fig. 3 .

The-first-power-of-the-diameter law is valid for the evaporation of a single drop on a heated plate [24]: $D_{0}^{2}-D^{2}=k t$, where $D$ is the base diameter of the drop at the time $t ; D_{0}$, its initial value; and $k$, the evaporation constant. One can classify the time
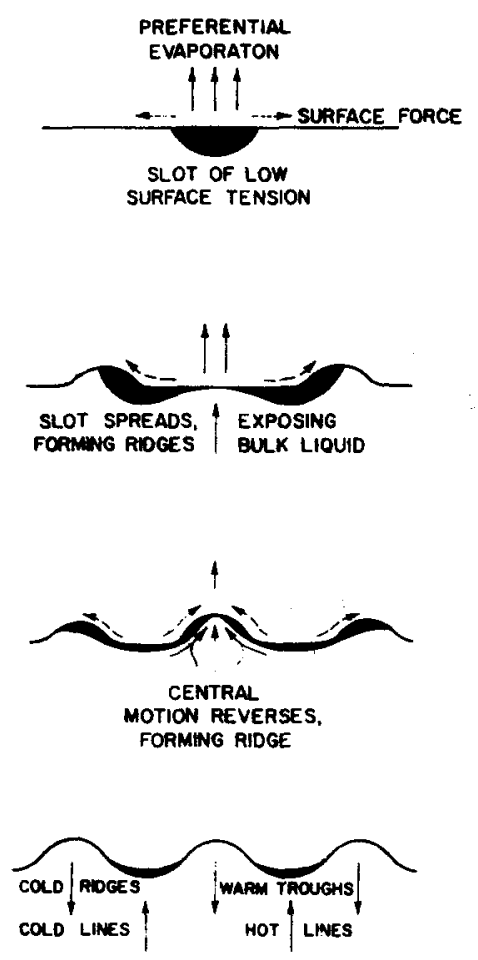

FIG. 3

A Model for the Mechanism of Interfacial Turbulence in a Minute Drop Fvaporating on a Solid Surface 
history of drop evaporation into three stages according to the rate of surface regression $\mathrm{dD} / \mathrm{dt}=\mathrm{k} /\left[2\left(\mathrm{D}_{0}^{2}-\mathrm{kt}\right)^{1 / 2}\right]$ which varies from $\mathrm{k} /\left(2 \mathrm{D}_{0}\right)$ to infinity: In the initial stage of evaporation where surface regression is nearly constant at $k /\left(2 D_{0}\right)$, the interface is dominated by radial stripes. The intermediate stage that follows begins when the drop surface withdraws more rapidly with time, during which polygonal cells appear in the interface. Finally, ripples encompass the interface in the asymptotic stage of evaporation where the surface regression rate is extremely high.

In the case of condensation, heat transfer causes an increase in surface tension. Hence a localized spot of preferential condensation has a higher surface tension than its surrounding interface liquid. This spot has no tendency to spread. With no motion being induced, there is no ripple formation and therefore the interface remains calm.

\section{Concluding Remarks}

Three fundamental structural forms of interfacial flow, radial stripes, polygonal cells and ripples, are disclosed and related to three stages of drop evaporation. The mechanism of ripple formation is proposed as caused by the liquid-air interface surface tension changes in a negative direction. The effect of fluid properties on interfacial turbulence is discussed. The study provides some insight into interfacial activities in drop evaporation on a plate. However, effort must be continued, in particular, to investigate the wave characteristics of interfacial turbulence such as the wavelength and speed of propagation.

\section{Acknowledgement}

The research work was supported by the National science Foundation under Grant No. ENG-7816972. The advice of Professor c. M. Vestin setting up the optical system is gratefully acknowledged. 


\section{References}

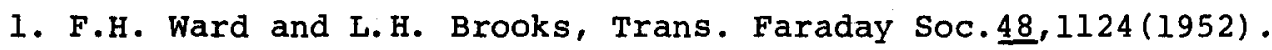

2. C.V. Sternling and L. E. Scriven, AIChE J. $\underline{5}, 514$ (1959).

3. A. Orell and J.W. Westwater, Chem. Eng. Sci. 16, 127 (1961).

4. A. Orell and J.W. Westwater, AIChE J. 8,350 (1962).

5. S. R. M. Ellis and M. Biddulph, Chem. Eng. Sci. 21,1107(1966).

6. P.L. T. Brian,J. E. Vivian and D.C. Matiatos,AIChE J. 31,28 (1967) .

7. E. Ruckenstein and C. Berbente,Chem. Eng. Sci. 19, 329 (1964).

8. D.A. Haydon, Proc. Roy. Soc. A 243, 483 (1958).

9. T.V. Davis and D.A. Haydon, ibid., 492

10.A. Vidal and A. Acrivos, Ind. \& Eng. Chem.-Fund. 2,53 (1968).

11.A. Vidal and A. Acrivos, J. Fluid Mech. 26, 809 (1966).

12.J.C. Berg,M. Boudart and A. Acrivos, Evaporative Cooling, Advances in Chemical Engineering, vol.6, 61 (1966).

13.J.C.Berg,M. Boudart and A.Acrivos,J.Fluid Mech. 24,721 (1966).

14.D. B. R. Kenning, Appl. Mech. Rev. 21, 1101 (1968) .

15. Wen-Jei Yang,Vaporization and Combustion of Liquid Drops on Heated Surfaces, in T.N. Veziroglu and S. Kakac (eds.) TwoPhase Transport and Reactor Safety, vol.1, Hemisphere 51(1976).

16.A. Moriyama, Trans. Iron \& Steel Inst. Japan 14, 285 (1974).

17.A. Moriyama, ibid., 290.

18.J.J. Lorenz and B.B. Mikic, J. Heat Transfer 92, 46 (1970).

19.B. K. Larkin, AIChE J. 16101 (1970).

20.J.C. Han and Wen-Jei Yang, J. Heat Transfer 102, 58 (1980).

21.J.D. Trolinger, Laser Applications in Flow Diagnostics, AGARDograph No. 186, NATO, chap. I (1974).

22.W. Merzkirch, Flow Visualization, Academic .Rress, New York (1974).

23.W. Hauf and U. Grigull, Optical Methods in Heat Transfer, in J. P. Hartnett and T.F. Irvine (eds.) Advances in Heat Transfer, vo1. 6, 133,Academic Press, New York (1970).

24.Z. Tamura and $Y$. Tanasawa, Evaporation and Combustion of a Drop Contacting with a Hot Surface, Proc.7th Symp.on Comb.509(1950). 